\title{
Editorial
}

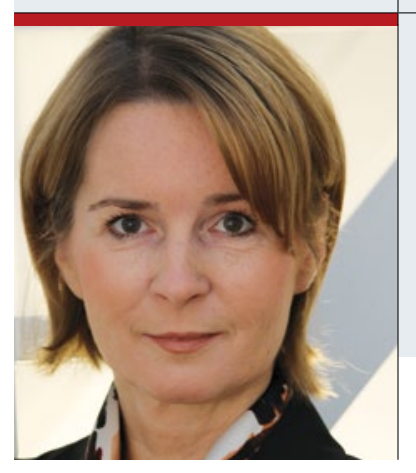

Katja Kupfer-Geißler

Chefredaktion

katja.kupfer@springer.com

facebook.com/springerpflege

\section{Selbst und ständig}

$\mathrm{T}$ räumen Sie auch manchmal von der Selbstständigkeit? Anlässe gäbe es ja genug: Frust auf Station, der Wunsch nach mehr Geld am Monatsende auf dem Konto oder eine freiere Zeiteinteilung. Ist aber auch ein Wagnis. Schließlich trägt man als Selbstständiger alle unternehmerischen Risiken und muss Auftragsflauten aber auch die fehlende Lohnfortzahlung im Krankheitsfall aushalten können. Bernd Welk, der Pfleger auf unserem Titelbild, hat es gewagt. Er ist den Schritt in die Freiberuflichkeit gegangen. Ein Schritt, den er nicht bereut, wie er Jörg Schmal berichtet hat (ab Seite 54).

Vor gut zwei Jahren berichtete Raffael Schmidt über die führende Rolle der Pflege bei der Implementierung eines Case Managements am Immanuel Klinikum. Ziel war es seinerzeit, ein optimales Prozessmanagement zu entwickeln, um Brüche an den Schnittstellen zum externen Versorgungssys- tem zu vermeiden. Jetzt hat man sich in Bernau das Belegungsmanagement vorgenommen. Über das Pilotprojekt und die gelungene Bewährungsprobe berichtet er nun in seinem Beitrag "Zur richtigen Zeit im richtigen Bett?" (Seite 48).

Und dann haben wir noch interessante PflegeKollegs für Sie: „Nadelstichverletzungen: Was tun?“ und „Inkontinenz: Richtig handeln“.

\section{Viel Freude beim Fortbilden mit HEILBERUFE} Ihre

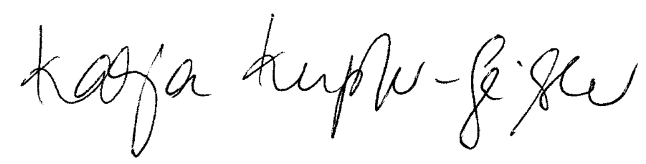

Anregungen, Wünsche, Fragen oder Lob?

Schreiben Sie uns: katja.kupfer@springer.com

\section{INFO}

\section{Willkommen in Dresden}

Auf dem 5. Interprofessionellen Gesundheitskongress (28. und 29. April in Dresden) von Springer Pflege stellt Evelyn Möhlenkamp, Generalbevollmächtigte Klinisches Prozessmanagement bei der Kliniken Südostbayern AG, die Schritte zu einem Lean Hospital vor. Weitere Schwerpunkte des Kongresses, zu dem rund 800 Teilnehmer erwartet werden, sind Ethik im Gesundheitswesen sowie Demenz im Akutkrankenhaus. Unter dem Leitspruch "Viele Professionen - ein Patient" steht dabei immer die Zusammenarbeit aller beteiligten Berufsgruppen im Zentrum der Diskussionen. Allerdings finden auch zahlreiche Seminare speziell für einzelne Berufsgruppen statt: Pflege Spezial, Medizin Spezial, MFA Spezial und Notfall Spezial.

Auch in diesem Jahr ergänzen das HeilberufeSCIENCE-Symposium mit Posterpräsentation und ein Schülertag von Auszubildenden für Auszubildende das Programm.

Wir freuen uns auf Sie!

\# IntGeKo2017

www.gesundheitskongresse.de 